\title{
Molecular Cloning and Characterization of Tropomyosin, a Major Allergen of Chironomus kiiensis, a Dominant Species of Nonbiting Midges in Korea
}

\author{
Kyoung Yong Jeong, ${ }^{1}$ Hye-Yung Yum, ${ }^{2}$ In-Yong Lee, ${ }^{1}$ Han-Il Ree, ${ }^{1}$ Chein-Soo Hong, ${ }^{3}$ \\ Dong Soo Kim, ${ }^{4}$ and Tai-Soon Yong ${ }^{1 *}$ \\ Department of Parasitology and Institute of Tropical Medicine, ${ }^{1}$ Department of Internal Medicine and Institute of \\ Allergy, ${ }^{3}$ and Department of Pediatrics, ${ }^{4}$ Yonsei University College of Medicine, Seoul, and Department of \\ Pediatrics, College of Medicine, Pochon CHA University-CHA General Hospital, \\ Pochon-gun, ${ }^{2}$ South Korea
}

Received 11 September 2003/Returned for modification 3 November 2003/Accepted 21 November 2003

\begin{abstract}
Chironomids are widely and abundantly distributed in the vicinity of standing waters. Larvae of Chironomus and some other genera are known to contain hemoglobins, which have been described as a major allergen, and the adults that have no hemoglobins also have been reported to contain allergens. In this study, we tried to establish the role of chironomid allergy and characterize the allergen of Chironomus kiiensis adults. Skin tests using $C$. kiiensis adult extracts were performed on patients with allergic symptoms. A cDNA library of $C$. kiiensis adults was screened with $C$. kiiensis immune mouse sera to identify allergens, and results were confirmed using skin test-positive human sera. Recombinant allergen was expressed in Escherichia coli and purified by affinity chromatography using nickel-nitrilotriacetic acid agarose to investigate its allergenic properties. Out of 275 allergic patients $\mathbf{1 4 . 2 \%}$ showed a positive reaction to $C$. kiiensis adult crude extracts in the skin test. The tropomyosin was cloned by immunoscreening and expressed in Escherichia coli. C. kiiensis tropomyosin has a high homology at the amino acid level with tropomyosins which were previously known to be allergens in various arthropods (Periplaneta americana, 86.3\%; Panulirus stimpson, 78.9\%; Dermatophagoides pteronyssinus, 76.5\%). Specific immunoglobulin $\mathrm{E}$ antibodies reacting to recombinant tropomyosin were detected in $17(81 \%)$ of 21 patients whose skin test results were positive. Cross-reactivity against house dust mites and other insects was noticed with mouse anti-recombinant tropomyosin immune serum. $C$. kiiensis adults were shown to be an important source of inhalant allergens in Korea. Molecular cloning of $C$. kiiensis tropomyosin was performed and IgE reactivity was demonstrated using skin test-positive human sera. Recombinant tropomyosin will be useful for further studies or clinical applications.
\end{abstract}

Adults and larvae of nonbiting midges are known to have potent allergens that cause immunoglobulin E ( $\mathrm{IgE})$-mediated asthma or, less frequently, allergic conjunctivitis. Allergy nomenclature in this paper follows the revised version (7). It has been reported that Cladotanytarsus lewisi occurred in enormous numbers in the Nile basins in Sudan and caused severe respiratory diseases in people living close to the Nile (10). Chironomid larvae were widely used as fish food in Germany and elicit occupational allergic diseases in a significant number of people who were exposed to these larvae $(1,15)$.

Hemoglobins, which are unique components in chironomid larvae among insects, have been identified as the most important allergen found in asthmatic patients $(2,3,9,18,28)$. On the other hand, $40 \%$ of Japanese, Taiwanese, and Swedish sera from patients with IgE-mediated asthma showed positive reactions against crude extracts of a midge (Cricotopus sylvestris) extract which contained no hemoglobin (29). Up to the present, several clinical cases in which patients had positive skin tests or positive results for chironomid-specific $\operatorname{IgE}$ have been reported. Few studies on chironomid allergens except for he-

\footnotetext{
* Corresponding author. Mailing address: Department of Parasitology and Institute of Tropical Medicine, Brain Korea 21 Project for Medical Science, Yonsei University College of Medicine, Seoul 120 752, Korea. Phone: 82-2-361-5290. Fax: 82-2-363-8676. E-mail: tsyong212 @yumc.yonsei.ac.kr.
}

moglobin have been carried out, although a protein with a high molecular weight in the whole-body extract of chironomid adults has been suggested to be more allergenic than hemoglobin (16).

People are unlikely to inhale the hemoglobin of chironomid larvae, which live under water, but the dead debris of ephemeral chironomid adults, which often swarm and gather around lights, is more likely to contact persons and cause allergic diseases.

Chironomus kiiensis and Chironomus flaviplumus were reported to be the most prevalent species of chironomids in Korea out of about 50 recorded species to date (20). The latter emerges from eutrophic waters in urban areas, whereas the former is distributed mainly in rural areas, breeding in rice paddies, lakes, and other clean waters.

In the present study, IgE immunoblot analyses were performed using both human sera from individuals who had positive skin test reactions to the chironomid extract and the immunized BALB/c mouse sera in order to identify IgE-binding proteins in whole-body extracts of $C$. kiiensis adults, which represent the dominant species of nonbiting midges in Korea.

\section{MATERIALS AND METHODS}

Collection of $\boldsymbol{C}$. kiiensis and preparation of the crude extract. C. kiiensis adults were collected every three to four days per week from July to October in 1997 using three light-traps on rice paddies at Dokyang-gu, Koyang-si, Kyonggi-do, 
Korea. C. kiiensis adults were identified following the key for morphological identification and stored at $-70^{\circ} \mathrm{C}$ until used (20).

Frozen midges were defrosted, homogenized, and then extracted in $10 \mathrm{mM}$ phosphate-buffered saline, $\mathrm{pH} 7.4$, by incubation for $10 \mathrm{~h}$ at $4^{\circ} \mathrm{C}$. The extracts were used for the enzyme-linked immunosorbent assay or immunoblot analysis. Lyophilized midges were extracted in the modified Coca solution $(0.9 \% \mathrm{NaCl}$, $0.25 \% \mathrm{NaHCO}_{3}, 0.04 \%$ phenol), and 1:40 (wt/vol) diluted extract at a final concentration of $1.5 \mathrm{mg} / \mathrm{ml}$ was used for the skin prick test (19). The tip of a 26-gauge needle was introduced through a drop of the extract into the superficial layers of the skin, which were gently lifted. A reaction was considered positive when a wheal with a diameter of $3 \mathrm{~mm}$ developed. Positive (histamine dihydrochloride [1 mg/ml]; Bayer) and negative (albumin-saline with phenol; Bayer) controls were applied in all tests.

Skin prick test and collection of human sera. The skin prick test using C. kiiensis crude extracts was performed on patients who came to the allergy clinic of the Severance Hospital, Yonsei University College of Medicine, Seoul, Korea. A total of 275 subjects, which included females 10 to 75 years old ( $n=$ 138; average age, 41 years) and males 15 to 69 years old ( $n=147$; average age, 31 years), were enrolled in the study. All subjects underwent the skin prick test using extracts of Dermatophagoides pteronyssinus, Dermatophagoides farinae, Blattella germanica, Periplaneta americana, C. kiiensis, Momomorium pharaonis, Drosophila melanogaster, Penicillium mix, and Aspergillus fumigatus (Bencard Co., Gaggenau, Germany). Sera of the skin test-positive subjects were collected from September 1998 to February 1999 and kept frozen at $-20^{\circ} \mathrm{C}$.

Production of antisera against $\boldsymbol{C}$. kiiensis adult crude extract in mice. Three 6-week-old female BALB/c mice were immunized to obtain antiserum. Aluminum hydroxide gel (alum) was prepared as described previously (21). Twenty micrograms of the crude extract in phosphate-buffered saline was mixed with 1 $\mathrm{ml}$ of alum, and the mice received intraperitoneal injections with the crude extract-gel ( $0.1 \mathrm{ml}$, containing $2 \mu \mathrm{g}$ of the crude extract and about $14 \mathrm{mg}$ of alum) at $0-, 4-$, and 8-week intervals.

Construction of a cDNA expression library. The frozen sample of $C$. kiiensis after morphological identification was homogenized in liquid nitrogen and mRNA was purified by using the mRNA isolation kit (Stratagene, La Jolla, Calif.), and a cDNA expression library in the ZAP Express vector (Stratagene) was constructed using a ZAP Express cDNA Gigapack II gold cloning kit (Stratagene) according to the manufacturer's instruction. Immunoscreening was carried out by phage dot immunoblot analysis using a picoBlue immunoscreening kit (Stratagene) with a 1:500 dilution of serum from mice immunized with crude extract of $C$. kiiensis and alkaline phosphatase-labeled anti-mouse IgG1. Positive clones were rescreened until all plaques on the plate were positive. Selected clones in ZAP Express vector were excised in vivo with ExAssist helper phages (Stratagene).

DNA sequencing and homology search. DNA sequencing of plasmid purified from the transformed bacteria was performed by the dideoxy nucleotide chain termination method (26) using a Sequenase version 2.0 DNA sequencing kit (Amersham Life Science, Cleveland, Ohio). Analysis of the derived nucleotide and deduced amino acid sequences was performed using the NCBI/BLAST network service of the National Center for Biotechnology Information, Bethesda, Md. A homology search in GenBank was performed using the NCBI Blast program.

Production of the recombinant tropomyosin. Tropomyosin-encoding cDNA was subcloned into the Bam $\mathrm{HI}$ and $\mathrm{XhoI}$ sites of the expression vector pET-28a and expressed in Escherichia coli BL21(DE3). The cells were grown to an optical density at $600 \mathrm{~nm}$ of 0.6 in Luria-Bertani broth (23) containing kanamycin (50 $\mu \mathrm{g} / \mathrm{ml})$, and then expression was induced by the addition of isopropyl-thio- $\beta$ galactoside to a final concentration of $1 \mathrm{mM}$. Incubation was continued for another $5 \mathrm{~h}$, and the cells were harvested by centrifugation. Recombinant tropomyosin was purified by affinity column chromatography using Ni-NTA Agarose (QIAGEN, Valencia, Calif.) according to the manufacturer's manual.

IgE immunoblot analysis. Proteins after sodium dodecyl sulfate (SDS)-polyacrylamide gel electrophoresis were electrophoretically transferred onto the nitrocellulose membranes and reacted with 1:2-diluted human sera overnight at room temperature. After washing five times, the membranes were incubated with 1:1,000-diluted monoclonal (clone GE-1) anti-human IgE alkaline phosphatase conjugate (catalog no. A-3076; Sigma, St. Louis, Mo.) for $1 \mathrm{~h}$ at room temperature. Color was developed with 3-bromo-4-chloro-5-indolyl-phosphate (758 ng/ $\mathrm{ml})$ and nitroblue tetrazolium $(379 \mathrm{ng} / \mathrm{ml})$ in a solution containing $100 \mathrm{mM}$ Tris-Cl (pH 9.5), $100 \mathrm{mM} \mathrm{NaCl}$, and $5 \mathrm{mM} \mathrm{MgCl}_{2}$.

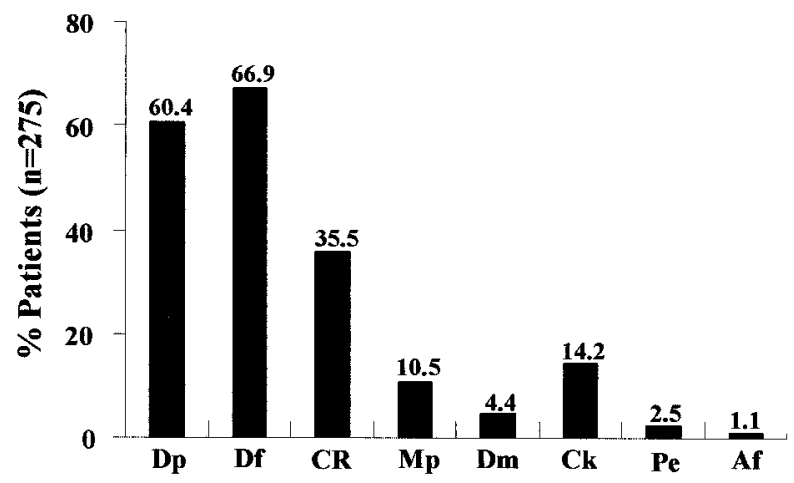

FIG. 1. Skin test reactivity to respiratory allergens among patients. Abbreviations: Dp, D. pterronyssis; Df, D. farinae; CR, P. americana and B. germanica; Mp, M. pharaonis; Dm, D. melanogaster; Ck, C. kiiensis; Pe, Penicillium; Af, A. fumigatus.

\section{RESULTS}

Frequency of chironomid allergy among allergic patients in Korea. Patients complaining of allergic asthma, allergic rhinitis, allergic urticaria, allergic conjunctivitis, throat irritation, atopic eczema/dermatitis syndrome, or itching underwent the skin prick test (Fig. 1). Thirty-nine of 275 subjects with the symptoms of allergic asthma, allergic rhinitis, allergic urticaria, or throat irritation showed a positive reaction to the $C$. kiiensis crude extract. As shown in Fig. 1, house dust mites are the most potent elicitor of allergic disease, and cockroaches are the second.

Cloning of $\boldsymbol{C}$. kiiensis tropomyosin. Six strongly positive clones were identified by immunoscreening with serum from mice immunized with $C$. kiiensis crude extract, and all clones were found to have the identical cDNA sequence.

The cloned cDNA sequence contains an 855-bp open reading frame coding for 285 amino acids, with an estimated molecular mass of $32,531 \mathrm{Da}$ and a $\mathrm{pI}$ of 4.58. No potential $\mathrm{N}$-linked glycosylation sites were found (Fig. 2). As shown in Fig. 3, BLAST search showed that $C$. kiiensis tropomyosin has the highest homology with insect tropomyosins (D. melanogaster, 89.1\%; Periplaneta americana, 86.3\%). It also has high sequence identities with those of two other major arthropod groups that are known to be major allergens, such as Panulirus stimpson (Crustacea, 78.9\%) and D. pteronyssinus (Chelicerata, $76.5 \%$ ). This sequence, which was deposited in the GenBank under accession number AJ012184, was designated as Chi k 10, according to the allergen nomenclature of the World Health Organization and the International Union of Immunological Societies (13).

Purification of recombinant tropomyosin. The purified recombinant tropomyosin containing 34 more amino acids at the $\mathrm{N}$ terminus gave a band on Coomassie blue-stained SDS-polyacrylamide gels with an apparent molecular mass of $45 \mathrm{kDa}$. The size is larger than the one calculated from the nucleotide sequence. The mobility of the tropomyosin significantly reduced in the presence of $6 \mathrm{M}$ urea, giving an apparent molecular mass of $67.6 \mathrm{kDa}$, whereas ovalbumin migrated at the expected position (Fig. 4). The final yield of the purified recombinant protein measured by Bradford assay (Bio-Rad, Hercules, Calif.) was approximately $2 \mathrm{mg} /$ liter of $E$. coli culture. 
1 cgcggat tcagat tcaagtt t t acagt t gagt at t taat tgtgaaagt tatcgaat aa

61 atctgccaacatggatgccatcaagaaaaagatgcaagcgat gaagct tgagaaggat aa $\begin{array}{llllllllllllllllllllllllllll}M & D & A & I & K & K & K & M & Q & A & M & K & L & E & K & D & N\end{array}$

121 tgcettagatcgtgccettctctgtgaaatcaagctcgtgatgctaact tgcgtgctga

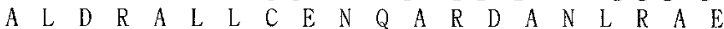

181 aaaggct gaagaagaagccegcacat tgcaaaagaagat ccaaact at tgaaacgat t

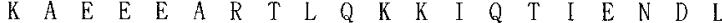
241 ggatcaaacacaaggacaagaaact ct tgtcaatggcaaactcgaaggaaaggaaaaggc

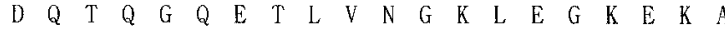
301 tctccaaaatgctgaatcagaagt cgctgct t tgaaccgtcgtatccaat tgt gggaga

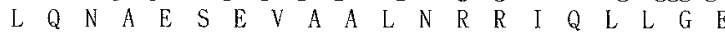
361 agatct tgaccgt tcagaagaacgt t ggct tcagcaacagcaaaat tgt cagaagcatc

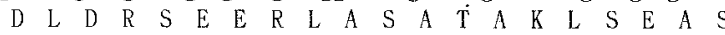
421 agctgctgctgatgagtctgaacgtgcacgt aaaat tct tgaaaat cggtcactcgctga $\begin{array}{llllllllllllllllllllllllllllllll}A & A & A & D & E & S & E & R & A & R & K & I & L & E & N & R & S & L & A & D\end{array}$ 481 tgaagaacgtatggatgccetcgaaaatcaat tgaaggaagcacgct tcct tgcagaaga

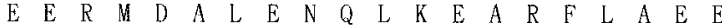
541 agcagacaaaaaat acgatgaggt tgcacgt aaat ggct atggt tgaggct gat ct ${ }^{\text {ga }}$

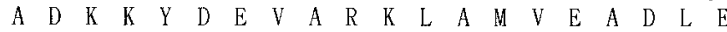
601 acgtgctgaagaacgtgcagaggct ggtgaagct aaaat tgt tgaact tgaagaagaact

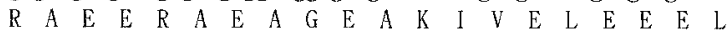
661 ccgtgt tgtaggcaacaact tgaaatcat tagaagt ct cagaagaaaaggcaaaccaacg

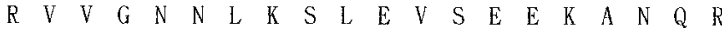

721 tgaagaagaatacaaaatcaaatcaaaacctcacaaccegtcttaaggaggctgaagc $\begin{array}{llllllllllllllllllll}E & E & E & Y & K & N & Q & I & K & T & L & T & T & R & L & K & E & A & E & A\end{array}$ 781 ccgcgcagaat tcgct gaacgt tccgt tcagaaat gcagaaggaagtcgacagact tga

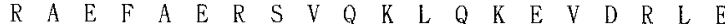
841 agacgaactcgt ttccgaaaaggaaaagt acagagaaat tggagacgatctcgacaccgc

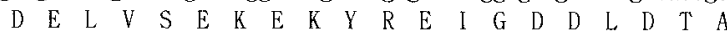
901 at tcgtggaact tatcctcaagaataagcaaatet tcatcgcaaat caaaatggaaatg F V E L I L K E

961 agcact tatggatatt ttctat tat tctcaaaat at tcat tgt tataaaat tccgtc 1021 ttttact tactacaaaaaacacacacacacacaaat cactaat ggatcacctacaa

1081 atgatactt tataatt tcatt ttgactattat tcactcaattt taattcaatccac

1141 atgtaat tat aacggt taagt tat aaaaaat tatcat at ttcct taaacctgtgcc

1201 t tgaactgaaacat caacaaggaacat act $t \mathrm{t}$ taat gaaactgcagat tgataat

1261 t t t aaaacaact t acgatcgaaat t tgtcgt tt tt ttgtt tat t at tat tat at

1321 ggt tgaaacat acat t caatgat gt cgt agt cgagaat at t t gcaaacat at $t$ t ta

1381 tggaat aaagggaaagaagt t t at tat tgaacatt t aaaaaaaaaaaaaaaaaaa 1441 a

FIG. 2. Nucleotide and deduced amino acid sequences of $C$. kiiensis tropomyosin. The estimated molecular mass is $32.531 \mathrm{kDa}$, and no potential N-linked glycosylation sites were identified.

IgE immunoblot analysis. Immunoblot analysis was carried out to confirm the reactivity of the recombinant protein. IgEreacting antibodies to the recombinant tropomyosin were detected in $17(81 \%)$ of 21 sera tested (Fig. 5). Serum from the nonallergic control was not reactive to the recombinant protein.

\section{DISCUSSION}

Two cases of asthmatic patients allergic to Chironomus plumosus and Tokunagayusurika akamusi extracts were reported in Korea (11). Twenty percent of the Korean patients with respiratory allergic symptoms were shown to react positively with chironomid extracts (19). Since the two species of chironomids that were used in these experiments are rarely found in Korea, the results indicate that their chironomid antigens contained materials cross-reactive to other chironomid species which are widely distributed in Korea. A high frequency of $\operatorname{IgE}$ antibody responses to chironomids was also demonstrated in Japanese patients with allergic asthma (14).

Chironomid allergy accounts for $14.2 \%$ of allergic subjects, which is more than the proportion of other allergic diseases that are caused by known indoor allergens except those caused by house dust mites and cockroaches. Large numbers of patients are likely to be found in the vicinity of standing waters where nonbiting midges emerge in large numbers. High levels of chironomid antigens in both air and soil were demonstrated, and a seasonal fluctuation in the air which peaked only in the autumn was also examined (12).

We have previously reported that the various bands of 110 , $80,73,46,40,37$, and $34 \mathrm{kDa}$ of the chironomid adult extracts were found to react with skin test-positive human sera by $\operatorname{IgE}$ immunoblot analysis (31).

Change of allergenicity during metamorphosis was investigated. Mature adult extracts were shown to contain more substances that bound to the $\mathrm{IgE}$ of those chironomid allergic subjects $(16,17)$. Hemoglobin, known as a major allergen of chironomids $(4,27,30)$, is known to be degraded during metamorphosis $(17,24)$. Galindo et al. have reported that subjects unexposed to midges could have IgE to chironomid hemoglobin (5) and hypersensitivity to these larvae without apparent contact with them (6). This led us to suppose that a different kind of allergen could be found in the adult. By using the IgG1
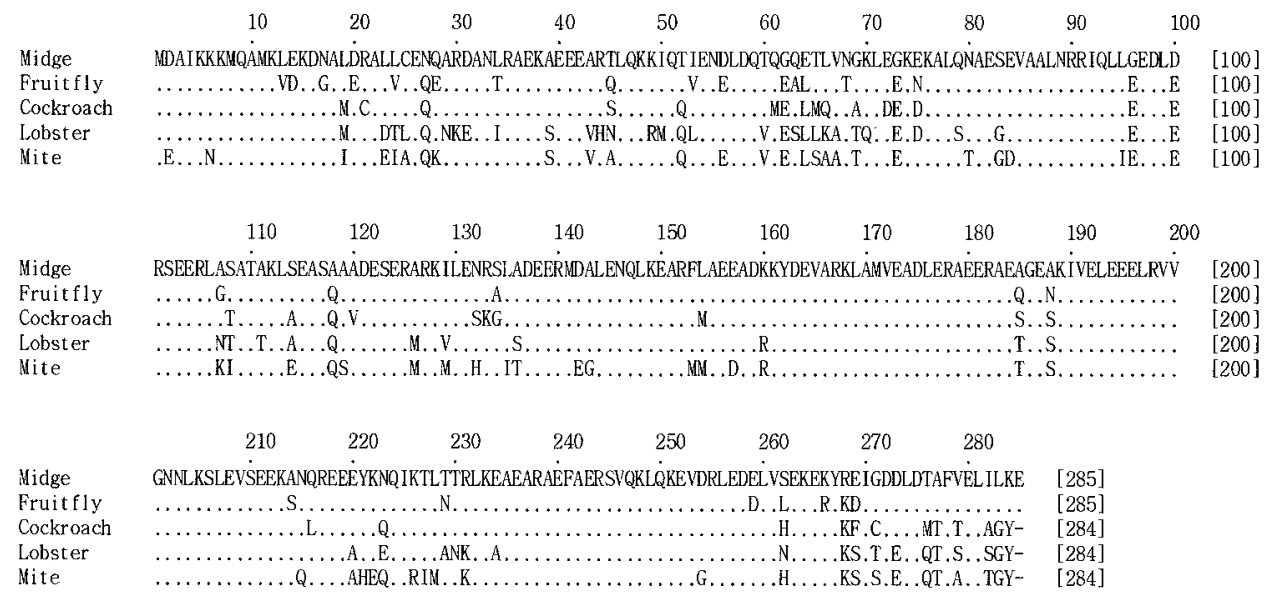

FIG. 3. Alignment of amino acid sequences of four arthropod tropomyosins: midge (C. kiiensis, accession number CAA09938), fruit fly ( $D$. melanogaster, accession number P43689), cockroach (P. americana, accession number CAB38086), lobster (P. stimpson, accession number AF030063), and mite (D. pteronyssinus, accession number Y14906). A dot indicates amino acid identity with $C$. kiiensis. 
(A)

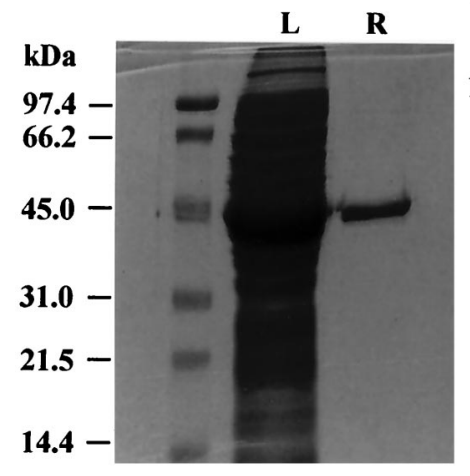

(B)

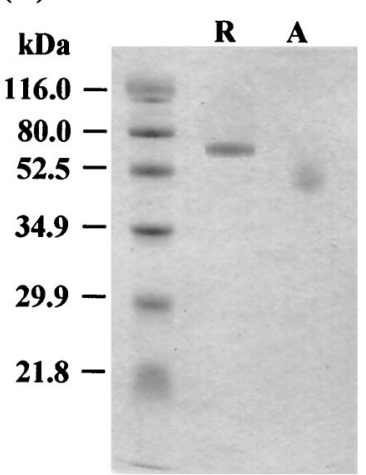

FIG. 4. Mass production of the recombinant allergen. (A) Recombinant tropomyosin was expressed in E. coli BL21(DE3) and purified by affinity column chromatography using Ni-NTA agarose. (B) Mobility of the recombinant allergen was tested in SDS-polyacrylamide gel containing $6 \mathrm{M}$ urea. Lanes: L, lysate of E. coli; R, recombinant tropomyosin; A, ovalbumin.

of mouse immune serum, which is known to be produced by the influence of interleukin-4, tropomyosin was cloned from the cDNA library of chironomid adults. Recombinant protein was expressed in bacteria to test the allergenicity. The change in migration on electrophoresis in SDS gels containing $6 \mathrm{M}$ urea is characteristic of tropomyosins (25). Tropomyosin-specific IgE was detected from 17 of 21 sera tested by immunoblotting, which indicated that tropomyosin is a major allergen of adult chironomid. Fifteen patients of 17 that were tropomyosin positive also had positive reactions to dust mites in the skin prick test. Of the 21 patients whose sera (19 patients were positive to dust mites) were used in IgE immunoblotting, only one patient had a positive reaction to $C$. kiiensis extract and negative reactions to mites and cockroaches. Five patients showed a stronger reaction (larger wheal size) to $C$. kiiensis than to mites and cockroaches in the skin test, implying the true sensitization of $C$. kiiensis tropomyosin. Only five subjects showed a stronger reaction to the mites and cockroaches. The other 10 persons showed similar reaction to the mites, cockroaches, and chironomid extract.

Tropomyosin, a kind of muscle protein, is a highly crossreactive allergen which sensitizes individuals who are genetically predisposed to food or house dust allergy (22). It is

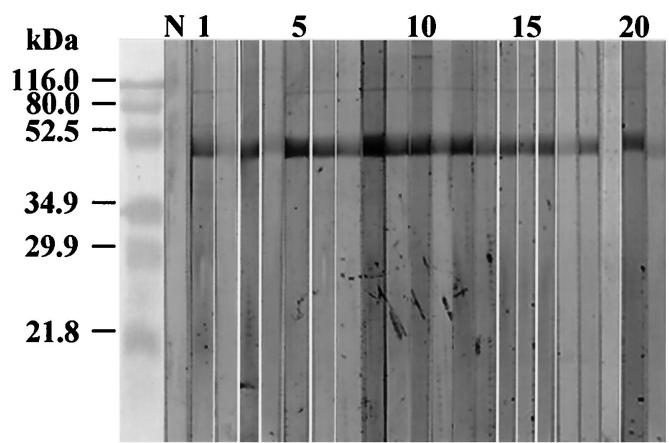

FIG. 5. IgE immunoblotting. Patients 2, 4, 17, 19, and 21 tested negative. Lanes: $\mathrm{N}$, negative control; 1 to 21 , sera of humans skin test positive to $C$. kiiensis extract. suggested that tropomyosin is an invertebrate pan-allergen because of its high cross-reactivity. In the immunoblot experiments, the bands of 70 to $80 \mathrm{kDa}$ are thought to be the dimers, and the bands of 30 to 50 are considered to be isoforms (data not shown). This pattern of tropomyosin bands is consistent with avian ones (8) and may implicate the potential crossreactivity of other arthropods.

In conclusion, we have identified tropomyosin from C. $\mathrm{ki}$ iensis as an important adult nonbiting midge allergen. Tropomyosins of invertebrates share a high degree of sequence identity. Further investigations are necessary to examine the possibility of cross-sensitization between allergic disorders caused by mites and insects. This recombinant allergen will be useful for clinical applications and allow a more detailed immunological characterization to be made.

\section{ACKNOWLEDGMENTS}

We are greatly indebted to Jongweon Lee for his skillful technical assistance.

This work was supported by the Brain Korea 21 Project for Medical Science, Yonsei University.

\section{REFERENCES}

1. Baur, X., M. Dewair, G. Fruhmann, H. Aschauer, J. Pfletschinger, and G. Braunitzer. 1982. Hypersensitivity to chironomids (non-biting midges): localization of the antigenic determinants within certain polypeptide sequences of hemoglobins (erythrocruorins) of Chironomus thummi thummi (Diptera). J. Allergy Clin. Immunol. 69:66-76.

2. Baur, X., H. Aschauer, G. Mazur, M. Dewair, H. Prelicz, and W. Steigemann. 1986. Structure, antigenic determinants of some clinically important insect allergens: chironomid hemoglobins. Science 233:351-354.

3. Cranston, P. S. 1988. Allergens of non-biting midges (Diptera: Chironomidae): a systematic survey of chironomid haemoglobins. Med. Vet. Entomol. 2:117-127.

4. Czisch, M., V. Liebers, R. Bernstein, X. Chen, X. Baur, and T. A. Holak. 1994. Conformations of peptide fragments comprising the complete sequence of component III of Chi $t$ I and their relationship to T-cell stimulation. Biochemistry 16:9420-9427.

5. Galindo, P. A., M. Lombardero, P. Mur, F. Feo, E. Gomez, J. Borja, R. Garcia, and D. Barber. 1999. Patterns of immunoglobulin E sensitization to chironomids in exposed and unexposed subjects. Investig. Allergol. Clin. Immunol. 9:117-122.

6. Galindo, P. A., F. Feo, E. Gomez, J. Borja, R. Melero, M. Lombardero, D. Barber, and R. G. Rodriguez. 1998. Hypersensitivity to chironomid larvae. Investig. Allergol. Clin. Immunol. 8:219-225.

7. Johansson, S. G. O., J. O. Hourihane, J. Bousquet, C. Bruijnzeel-Koomen, S. Dreborg, T. Haahtela, M. L. Kowalski, N. Mygind, J. Ring, P. van Cauwenberge, M. L. van Hage-Hamsten, and B. Wuthrich. 2001. A revised nomenclature for allergy. An EAACI position statement from EAACI nomenclature task force. Allergy 56:813-824.

8. Kardami, E., D. Montarras, and M. Fiszman. 1983. Fast and slow chicken muscles contain different $\alpha$ - and $\beta$-tropomyosins. Biochem. Biophys. Res. Commun. 110:147-154.

9. Kawai, K., H. Tagoh, K. Yoshizaki, G. Murakami, and A. Muraguchi. 1996. Purification and characterization of an allergenic monomeric hemoglobin from a chironomid distributed worldwide, Polypedium nubifer. Int. Arch. Allergy Immunol. 110:288-297.

10. Kay, A. B., E. L. Gad, M. O. Rab, J. Stewart, and H. H. Erwa. 1978. Widespread IgE-mediated hypersensitivity in Northern Sudan to the chironomid Cladotanytarsus lewisi. Clin. Exp. Immunol. 34:106-110.

11. Kim, Y. J., and H. S. Park. 1994. Skin reactivity and specific IgE antibody to two non-biting midges in Korea respiratory allergy patients. J. Korean Med. Sci. 9:21-28.

12. Kimura, K. Y., H. Matsuoka, and A. Ishii. 1990. ELISA inhibition method in detection of mite and chironomid antigens in environmental samples of dust, soil and air. Allergy 45:167-173.

13. King, T. P., D. Hoffman, H. Lowenstein, D. G. Marsh, T. A. E. Platts-Mills, and W. Thomas. 1994. Allergen nomenclature. Int. Arch. Allergy Immunol. 105:224-233.

14. Kino, T., J. Chihara, K. Fukuda, Y. Sasaki, Y. Shogaki, and S. Oshima. 1987. Allergy to insects in Japan. III. High frequency of IgE antibody responses to insects (moth, butterfly, caddis fly, and chironomid) in patients with bronchial asthma and immunochemical quantitation of the insect-related airborne particles smaller than 10 microns in diameter. J. Allergy Clin. Immunol. 79:857-866. 
15. Lieberis, V., M. Hoernstein, and X. Baur. 1993. Humoral immune response to the insect allergen Chi $t 1$ in aquarists and fish-food factory workers. Allergy 48:236-239.

16. Matsuoka, H., A. Ishii, and S. Noono. 1988. Detection of $\operatorname{IgE}$ antibodies to larvae and adults of chironomids by enzyme-linked immunosorbent assay. Allergy 43:425-429.

17. Matsuoka, H., A. Ishii, J. Y. Kimura, and S. Noono. 1990. Developmental change of chironomid allergen during metamorphosis. Allergy 45:115-120.

18. Mazur, G., W. M. Becker, and X. Baur. 1987. Epitope mapping of major insect allergens (chironomid hemoglobins) with monoclonal antibodies. J. Allergy Clin. Immunol. 80:876-883.

19. Park, H. S., N. S. Rhu, D. I. Cho, and J. W. Kim. 1991. Two cases of bronchial asthma induced by Chironomus plumosus and Tokunagayusurika akamusi. J. Korean Soc. Allergol. 11:362-367.

20. Ree, H. I. 1993. Breeding places of non-biting midges (Chironomidae, Diptera) in Korea. Korean J. Entomol. 23:169-176.

21. Ree, H. I., S. H. Lee, Y. K. Kim, S. Jeon, J. K. Chang, and Y. S. Kim. 1996 Identification and characterization of allergens of Chironomus falviplumus adults (Chironomidae, Diptera) in mice. Korean J. Parasitol. 34:35-47.

22. Reese, G., R. Ayuso, and S. B. Lehrer. 1999. Tropomyosin: an invertebrate pan-allergen. Int. Arch. Allergy Immunol. 119:247-258.

23. Sambrook, J., E. F. Fritsch, and T. Maniatis. 1989. Molecular cloning: laboratory manual, 2nd ed. Cold Spring Harbor Laboratory Press, Cold Spring Harbor, N.Y.

24. Schin, K., J. J. Poluhowich, T. Gamo, and H. Laufer. 1974. Degradation of haemoglobin in Chironomus during metamorphosis. J. Insect Physiol. 20: 561-571.

25. Smillie, L. B. 1982. Preparation and identification of $\alpha$ - and $\beta$-tropomyosins. Methods Enzymol. 85:231-241.

26. Tabor, S., and C. C. Richardson. 1987. DNA sequence analysis with a modified bacteriophage T7 DNA polymerase. Proc. Natl. Acad. Sci. USA 84:4767-4771.

27. Tautz, C., H. P. Rihs, A. Thiele, P. Zwollo, L. R. Freidhoff, D. G. Marsh, and X. Baur. 1994. Association of class II sequences encoding DR1 and DQ5 specificities with hypersensitivity to chironomid allergen Chi $t$ I. J. Allergy Clin. Immunol. 93:918-925.

28. van Kampen, V., W. M. Becker, Z. Chen, H. P. Rihs, G. Mazur, M. Raulf, V. Liebers, S. Isringhausen-Bley, and X. Baur. 1994. Analysis of B-cell epitopes in the N-terminal region of Chi t I component III using monoclonal antibodies. Mol. Immunol. 31:1133-1404.

29. van Kampen, V., V. Liebers, A. Cruppon, and X. Baur. 1994. Chironomidae hemoglobin allergy in Japanese, Swedish, and German populations. Allergy 49:9-12.

30. van Kampen, V., V. Liebers, I. Sander, Z. Chen, X. Baur, M. Raulf-Heimsoth, and F. W. Falkenberg. 2001. B-cell epitopes of the allergen Chi t 1.01: peptide mapping of epitopes recognized by rabbit, murine, and human antibodies. Allergy 56:118-125.

31. Yong, T. S., J. S. Lee, I. Y. Lee, S. J. Park, G. M. Park, H. I. Ree, J. W. Park, C. S. Hong, and H. S. Park. 1999. Identification of Chironomus kiiensis allergens, a dominant species of non-biting midges in Korea. Korean J. Parasitol. 37:171-179. 